Bull. Korean Math. Soc. 50 (2013), No. 1, pp. 275-283

http://dx.doi.org/10.4134/BKMS.2013.50.1.275

\title{
GLOBAL EXISTENCE OF WEAK SOLUTIONS FOR A LOGARITHMIC WAVE EQUATION ARISING FROM Q-BALL DYNAMICS
}

\author{
XiaOsen HaN
}

\begin{abstract}
In this paper we investigate an initial boundary value problem of a logarithmic wave equation. We establish the global existence of weak solutions to the problem by using Galerkin method, logarithmic Sobolev inequality and compactness theorem.
\end{abstract}

\section{Introduction}

In this paper we study the global existence of weak solutions for the initial boundary value problem

$$
\left\{\begin{array}{l}
u_{t t}-\Delta u+u-u \log |u|^{2}+u_{t}+|u|^{2} u=0,(x, t) \in \Omega \times(0, T), \\
u=0,(x, t) \in \partial \Omega \times(0, T) \\
u(x, 0)=u^{0}(x), u_{t}(x, 0)=u^{1}(x), x \in \Omega
\end{array}\right.
$$

where $\Omega$ is a smooth bounded domain in $\mathbb{R}^{3}, u$ is a complex scalar field. This model arises from the study of Q-ball dynamics in theoretical physics (see [18]). This type of problems have many applications in many branches of physics such as nuclear physics, optics and geophysics [5,9,19]. The model (1.1) is closely related to the following equation with logarithmic nonlinearity

$$
\left\{\begin{array}{l}
u_{t t}-\Delta u+u-\varepsilon u \log |u|^{2}=0,(x, t) \in \mathcal{O} \times(0, T), \\
u=0,(x, t) \in \partial \mathcal{O} \times(0, T), \\
u(x, 0)=u^{0}(x), u_{t}(x, 0)=u^{1}(x), x \in \mathcal{O},
\end{array}\right.
$$

where $\mathcal{O}$ is a finite interval $[a, b]$, the parameter $\varepsilon$ measures the force of the nonlinear interaction and the nonlinear effects in quantum mechanics are very

Received August 3, 2011; Revised January 25, 2012.

2010 Mathematics Subject Classification. 35L20, 35L70, 58G16.

Key words and phrases. logarithmic nonlinearity, global existence, logarithmic Sobolev inequality, logarithmic Gronwall inequality.

This work was supported by the National Natural Science Foundation of China under grant 11201118, Henan Basic Science and Frontier Technology Program Funds under grant 112300410054, and the Key Youth Teacher Foundation of Department Education of Henan Province under grant 2011GGJS-210. 
small. The problem (1.2) is a relativistic version of logarithmic quantum mechanics introduced in $[3,4]$ and can also be obtained by taking the limit $p \rightarrow 1$ for the $p$-adic string equation $[15,16,21,22]$.

It is easy to see that the problem $(1.2)$ can be see as one dimensional case of (1.1) without the first order derivative term and cubic term. In [12], by using compactness method Gorka obtained the global existence of weak solutions to the problem (1.2). In [2] Bartkowski and Gorka studied the corresponding Cauchy problem for $(1.2) \mathcal{O}=\mathbb{R}$ without boundary conditions. The global existence of weak solutions, classical solutions and the traveling wave were obtained.

The model (1.1) is introduced in [18] for studying the dynamics of Q-ball in theoretical physics. The logarithmic nonlinearity is of much interest in physics, since it appears naturally in inflation cosmology and supersymmetric filed theories, quantum mechanics and nuclear physics $[1,10,20]$.

The main difference between our work and [12] lies in: our problem is in three dimensional case and involves another nonlinear term $|u|^{2} u$; there is no restrictions on the coefficient of the logarithmic nonlinear term $u \log |u|^{2}$.

Recently in [18] a numerical study of the model (1.1) is given. However, there is no theoretical analysis for the problem. The purpose of this paper is to give a mathematical analysis for the problem (1.1). We mainly establish the global existence of weak solutions to the problem (1.1). This can be realized in a few steps. Firstly we write the problem in a weak version. Secondly we construct approximate solutions by the Galerkin method. Finally we prove the convergence of the sequence of the approximate solutions. To get a priori estimates of the approximate solutions, we employ the Gross logarithmic Sobolev inequality and logarithmic Gronwall inequality, which are fundamental here.

We also mention some related mathematical work involving the logarithmic term in the literature. In [7] Thierry and Alain establish the existence and uniqueness of a solution for the corresponding Cauchy problem (1.1) in $\mathbb{R}^{3}$ without the first order term and the cubic nonlinear term. There have been some works on the logarithmic Schrödinger equation (for example, see $[6,8,13$, 14]).

The rest of our paper is organized as follows. In Section 2 we state the main result. Section 3 is devoted to the proof of the main result.

\section{Main result}

We use the following notations throughout this paper: denote by $(\cdot, \cdot)$ the inner product in $L^{2}(\Omega)$ and by $\langle\cdot, \cdot\rangle$ the duality pairing between $H_{0}^{1}(\Omega)$ and $H^{-1}(\Omega),\|\cdot\|_{p}$ the $L^{p}$ norm. We also use $C$ to denote a universal positive constant may take different values in different places. We introduce the definition of weak solutions for the problem (1.1).

Definition 2.1. A function $u$ defined on $[0, T]$ is called a weak solution of the problem $(1.1)$ if $u \in C_{w}\left([0, T] ; H_{0}^{1}(\Omega)\right), u^{\prime} \in C_{w}\left([0, T] ; L^{2}(\Omega)\right), u(0)=$ 
$u^{0}, u^{\prime}(0)=u^{1}$ and $u$ satisfies

$(2.1)\left\langle u^{\prime \prime}(t), \phi\right\rangle+(\nabla u, \nabla \phi)+(u, \phi)+\left(u^{\prime}, \phi\right)-\left(u \log |u|^{2}, \phi\right)+\left(|u|^{2} u, \phi\right)=0$

for a.e. $t \in[0, T]$ and all test functions $\phi \in H_{0}^{1}(\Omega)$.

Our main results read as follows.

Theorem 2.1. Assume that $u^{0}(x) \in H_{0}^{1}(\Omega), u^{1}(x) \in L^{2}(\Omega)$. Then, the problem (1.1) admits global weak solution defined on $[0, T]$ for any $T>0$.

\section{Proof of Theorem 2.1}

In this section we carry out the proof of Theorem 2.1. The proof is based on Galerkin method. To proceed the proof we need the Gross logarithmic Sobolev inequality and the logarithmic Gronwall inequality. For the convenience of the reader we state the results here.

Lemma 3.1. Assume $v \in H_{0}^{1}(\Omega)$ and $\Omega$ is a bounded smooth domain in $\mathbb{R}^{3}$. Then, for any $a>0$, it holds that

$$
\int_{\Omega}|v|^{2} \log |v| \mathrm{d} x \leq\left(\frac{3}{4} \log \frac{4 a}{\mathrm{e}}\right)\|v\|_{2}^{2}+\frac{a}{4}\|\nabla v\|_{2}^{2}+\|v\|_{2}^{2} \log \|v\|_{2} .
$$

Proof. See [17].

Lemma 3.2. Assume that $w(t)$ is nonnegative, $w(t) \in L^{\infty}(0, T), w(0) \geq 0$, and it satisfies

$$
w(t) \leq w(0)+a \int_{0}^{t} w(s) \log [a+w(s)] \mathrm{d} s, t \in[0, T],
$$

where $a>1$ is a positive constant. Then we have

$$
w(t) \leq(a+w(0))^{\mathrm{e}^{a t}}, \quad t \in[0, T] .
$$

Proof. See $[7,11]$.

We use the standard Galerkin method to construct approximate solutions. Let $\left\{w_{j}\right\}_{j=1}^{\infty}$ be the eigenfunctions of the operator $A=-\triangle$ with zero Dirichlet boundary condition and $D(A)=H^{2}(\Omega) \cap H_{0}^{1}(\Omega)$. It is well-known that $\left\{w_{j}\right\}_{j=1}^{\infty}$ forms an orthonormal basis for $L^{2}(\Omega)$ as well as for $H_{0}^{1}(\Omega)$. Moreover, the linear span of $\left\{w_{j}\right\}_{j=1}^{\infty}$ is dense in $L^{q}(\Omega)$ for any $1 \leq q \leq 6$. Let $\mathcal{P}_{k}$ be the orthogonal projection of $L^{2}(\Omega)$ onto $V_{k}=$ the linear span of $\left\{w_{1}, \ldots, w_{k}\right\}, k \geq 1$. Let $u_{k}(t)=\sum_{j=1}^{k} g_{k, j}(t) w_{j}$ be an approximate solution to (1.1) in $V_{k}$. Then $u_{k}(t)$ verifies the following system of ODEs:

$$
\begin{aligned}
& \left\langle u_{k}^{\prime \prime}(t), w_{j}\right\rangle+\left(\nabla u_{k}(t), \nabla w_{j}\right)+\left(u_{k}, w_{j}\right)+\left(u_{k}^{\prime}(t), w_{j}\right) \\
& -\left(u_{k} \log \left|u_{k}\right|^{2}, w_{j}\right)+\left(\left|u_{k}\right|^{2} u_{k}, w_{j}\right)=0, \\
& u_{k}(0)=\mathcal{P}_{k} u^{0}, u_{k}^{\prime}(0)=\mathcal{P}_{k} u^{1},
\end{aligned}
$$


for $j=1, \ldots, k$. More specifically,

$$
u_{k}(0)=\sum_{j=1}^{k} u_{k, j}(0) w_{j}, u_{k}^{\prime}(0)=\sum_{j=1}^{k} u_{k, j}^{\prime}(0) w_{j},
$$

where

$$
u_{k, j}(0)=\left(u^{0}, w_{j}\right), u_{k, j}^{\prime}(0)=\left(u^{1}, w_{j}\right), j=1, \ldots, k .
$$

Obviously, $u_{k}(0) \rightarrow u^{0}$ strongly in $H_{0}^{1}(\Omega), u_{k}^{\prime}(0) \rightarrow u^{1}$ strongly in $L^{2}(\Omega)$ as $k \rightarrow \infty$. By using the Cauchy-Peano theorem, we know that the system (3.1)(3.2) admits a solution $g_{k, j}(t) \in C^{2}\left[0, T_{k}\right]$ for every $k \geq 1$ and some $T_{k}>0$. Then we can obtain an approximate solution $u_{k}(t)$ of the problem (1.1) over $\left[0, T_{k}\right]$.

Now we try to get the a priori estimate for the approximate solutions $u_{k}(t)$ of the problem (1.1).

Multiplying (3.1) by $g_{k, j}^{\prime}(t)$ and summing with respect to $j$ from 1 to $k$, we have

$$
\begin{aligned}
& \frac{\mathrm{d}}{\mathrm{d} t}\left[\frac{1}{2}\left\|u_{k}^{\prime}(t)\right\|_{2}^{2}+\frac{1}{2}\left\|\nabla u_{k}(t)\right\|_{2}^{2}+\left\|u_{k}(t)\right\|_{2}^{2}-\int_{\Omega}\left|u_{k}(t)\right|^{2} \log \left|u_{k}(t)\right| \mathrm{d} x+\frac{1}{4}\left\|u_{k}(t)\right\|_{4}^{4}\right] \\
& +\left\|u_{k}^{\prime}(t)\right\|_{2}^{2}=0 .
\end{aligned}
$$

Integrating (3.3) over $(0, t), 0<t \leq T_{k}$, we get

$$
\begin{aligned}
& \frac{1}{2}\left\|u_{k}^{\prime}(t)\right\|_{2}^{2}+\frac{1}{2}\left\|\nabla u_{k}(t)\right\|_{2}^{2}+\left\|u_{k}(t)\right\|_{2}^{2}-\int_{\Omega}\left|u_{k}(t)\right|^{2} \log \left|u_{k}(t)\right| \mathrm{d} x \\
& +\frac{1}{4}\left\|u_{k}(t)\right\|_{4}^{4}+\int_{0}^{t}\left\|u_{k}^{\prime}(s)\right\|_{2}^{2} \mathrm{~d} s \\
= & \frac{1}{2}\left\|u_{k}^{\prime}(0)\right\|_{2}^{2}+\frac{1}{2}\left\|\nabla u_{k}(0)\right\|_{2}^{2}+\left\|u_{k}(0)\right\|_{2}^{2}-\int_{\Omega}\left|u_{k}(0)\right|^{2} \log \left|u_{k}(0)\right| \mathrm{d} x+\frac{1}{4}\left\|u_{k}(0)\right\|_{4}^{4} \\
\leq & C_{0}-\int_{\Omega}\left|u_{k}(0)\right|^{2} \log \left|u_{k}(0)\right| \mathrm{d} x,
\end{aligned}
$$

where $C_{0}=C\left(\left\|u^{0}\right\|_{H^{1}(\Omega)},\left\|u^{1}\right\|_{L^{2}(\Omega)}\right)$ is a positive constant. To deal with the last term in (3.4), we use the elementary inequality

$$
\left|t^{2} \log t\right| \leq C\left(1+t^{3}\right), \quad \forall t>0,
$$

where $C>0$ is a positive constant. Then by (3.5) we have

$$
\begin{aligned}
\left.\left|-\int_{\Omega}\right| u_{k}(0)\right|^{2} \log \left|u_{k}(0)\right| \mathrm{d} x \mid & \leq C|\Omega|+C \int_{\Omega}\left|u_{k}(0)\right|^{3} \mathrm{~d} x \\
& \leq C\left(1+\left\|u_{k}(0)\right\|_{H_{0}^{1}(\Omega)}^{3}\right) \\
& \leq C\left(1+\left\|u^{0}\right\|_{H_{0}^{1}(\Omega)}^{3}\right) .
\end{aligned}
$$


Hence combining (3.4) and (3.6) gives

$(3.7) \leq C+\int_{\Omega}\left|u_{k}(t)\right|^{2} \log \left|u_{k}(t)\right| \mathrm{d} x$.

$$
\frac{1}{2}\left\|u_{k}^{\prime}(t)\right\|_{2}^{2}+\frac{1}{2}\left\|\nabla u_{k}(t)\right\|_{2}^{2}+\left\|u_{k}(t)\right\|_{2}^{2}+\frac{1}{4}\left\|u_{k}(t)\right\|_{4}^{4}+\int_{0}^{t}\left\|u_{k}^{\prime}(s)\right\|_{2}^{2} \mathrm{~d} s
$$

Now we use Gross Sobolev inequality in Lemma 3.1 to estimate the last term on the righthandside of (3.7) as follows

$$
\begin{aligned}
& \int_{\Omega}\left|u_{k}(t)\right|^{2} \log \left|u_{k}(t)\right| \mathrm{d} x \\
\leq & \left(\frac{3}{4} \log \frac{4 a}{\mathrm{e}}\right)\left\|u_{k}(t)\right\|_{2}^{2}+\frac{a}{4}\left\|\nabla u_{k}(t)\right\|_{2}^{2}+\left\|u_{k}(t)\right\|_{2}^{2} \log \|v\|_{2} .
\end{aligned}
$$

Inserting (3.8) into (3.7), we have

$$
\begin{aligned}
& \quad \frac{1}{2}\left\|u_{k}^{\prime}(t)\right\|_{2}^{2}+\left(\frac{1}{2}-\frac{a}{4}\right)\left\|\nabla u_{k}(t)\right\|_{2}^{2}+\left(1-\frac{3}{4} \log \frac{4 a}{\mathrm{e}}\right)\left\|u_{k}(t)\right\|_{2}^{2} \\
& \quad+\frac{1}{4}\left\|u_{k}(t)\right\|_{4}^{4}+\int_{0}^{t}\left\|u_{k}^{\prime}(s)\right\|_{2}^{2} \mathrm{~d} s \\
& \leq C+\left\|u_{k}(t)\right\|_{2}^{2} \log \left\|u_{k}(t)\right\|_{2} .
\end{aligned}
$$

By taking $a=\frac{1}{4}$ in (3.9) we obtain

$$
\begin{aligned}
& \left\|u_{k}^{\prime}(t)\right\|_{2}^{2}+\left\|\nabla u_{k}(t)\right\|_{2}^{2}+\left\|u_{k}(t)\right\|_{2}^{2}+\left\|u_{k}(t)\right\|_{4}^{4} \\
\leq & C\left(1+\left\|u_{k}(t)\right\|_{2}^{2} \log \left\|u_{k}(t)\right\|_{2}\right) .
\end{aligned}
$$

Noting that

we have

$$
u_{k}(t)=u_{k}(0)+\int_{0}^{t} u_{k}^{\prime}(s) \mathrm{d} s
$$

$$
\begin{aligned}
\left\|u_{k}(t)\right\|_{2}^{2} & \leq 2\left\|u_{k}(0)\right\|_{2}^{2}+2 T \int_{0}^{t}\left\|u_{k}^{\prime}(s)\right\|_{2}^{2} \mathrm{~d} s \\
& \leq 2\left\|u_{k}(0)\right\|_{2}^{2}+\max \{1,2 T\} \frac{1+C}{C} \int_{0}^{t}\left\|u_{k}^{\prime}(s)\right\|_{2}^{2} \mathrm{~d} s .
\end{aligned}
$$

Then it follows from (3.10) and (3.11) that

$$
\left\|u_{k}(t)\right\|_{2}^{2} \leq A+B \int_{0}^{t}\left\|u_{k}(s)\right\|_{2}^{2} \log \left\|u_{k}(s)\right\|_{2} \mathrm{~d} s,
$$

where

$$
A=2\left\|u_{k}(0)\right\|_{2}^{2}+\max \{1,2 T\}(1+C) T, B=\max \{1,2 T\}(1+C) .
$$

Noting $B \geq 1$, then by the logarithmic Gronwall inequality in Lemma 3.2, we get

$$
\left\|u_{k}(t)\right\|_{2}^{2} \leq(A+B)^{e^{B t}} \leq C_{T}
$$


Therefore from (3.10) and (3.13) we conclude that

$$
\left\|u_{k}^{\prime}(t)\right\|_{2}^{2}+\left\|\nabla u_{k}(t)\right\|_{2}^{2}+\left\|u_{k}(t)\right\|_{2}^{2}+\left\|u_{k}(t)\right\|_{4}^{4} \leq C_{T} .
$$

The estimate (3.14) implies that $T_{k}=T$ and

$$
u_{k} \text { is uniformly bounded in } L^{\infty}\left(0, T ; H_{0}^{1}(\Omega)\right) \text {, }
$$

$$
u_{k}^{\prime} \quad \text { is uniformly bounded in } L^{\infty}\left(0, T ; L^{2}(\Omega)\right) \text {. }
$$

By a standard discussion, we obtain

(3.17) $\quad u_{k}^{\prime \prime}$ is uniformly bounded in $L^{\infty}\left(0, T ; H^{-1}(\Omega)\right)$.

Hence we can infer from (3.14)-(3.16) that there exists a subsequence of $\left\{u_{k}\right\}$, still denoted by $\left\{u_{k}\right\}$, such that

$$
\begin{array}{ccc}
u_{k} \rightarrow u & \text { weakly star in } & L^{\infty}\left(0, T ; H_{0}^{1}(\Omega)\right), \\
u_{k}^{\prime} \rightarrow u^{\prime} & \text { weakly star in } & L^{\infty}\left(0, T ; L^{2}(\Omega)\right), \\
u_{k}^{\prime \prime} \rightarrow u^{\prime \prime} & \text { weakly star in } & L^{\infty}\left(0, T ; H^{-1}(\Omega)\right) .
\end{array}
$$

Then using (3.18)-(3.20) and Aubin-Lions lemma we have

$$
u_{k} \rightarrow u \quad \text { strongly in } \quad L^{2}\left(0, T ; L^{2}(\Omega)\right),
$$

which implies

$$
u_{k} \rightarrow u \quad \text { a.e. in }(0, T) \times \Omega .
$$

It follows from (3.22) that

$$
\begin{array}{r}
u_{k} \log \left|u_{k}\right|^{2} \rightarrow u \log |u|^{2} \quad \text { a.e. in }(0, T) \times \Omega, \\
\left|u_{k}\right|^{2} u_{k} \rightarrow|u|^{2} u \quad \text { a.e. in }(0, T) \times \Omega .
\end{array}
$$

Using (3.5) again we can estimate the logarithmic nonlinear term as

$$
\begin{aligned}
\left.\left.\int_{\Omega}\left|u_{k} \log \right| u_{k}\right|^{2}\right|^{2} \mathrm{~d} x & =4 \int_{\Omega}\left|u_{k}\right|^{2}\left(\log \left|u_{k}\right|\right)^{2} \mathrm{~d} x \\
& \leq C|\Omega|+C \int_{\Omega}\left|u_{k}\right|^{6} \mathrm{~d} x \\
& \leq C\left(\left\|u_{k}\right\|_{H_{0}^{1}(\Omega)}^{6}+1\right) \\
& \leq C .
\end{aligned}
$$

That is to say,

$$
u_{k} \log \left|u_{k}\right|^{2} \quad \text { is uniformly bounded in } L^{\infty}\left(0, T ; L^{2}(\Omega)\right) .
$$

Then there exists some function $\chi \in L^{\infty}\left(0, T ; L^{2}(\Omega)\right)$ such that

$$
u_{k} \log \left|u_{k}\right|^{2} \rightarrow \chi \quad \text { weakly star } L^{\infty}\left(0, T ; L^{2}(\Omega)\right) .
$$

In view of (3.23) and Lemma 3.1.5 in [23], we have

which implies

$$
\chi=u \log |u|^{2}
$$

$$
u_{k} \log \left|u_{k}\right|^{2} \rightarrow u \log |u|^{2} \quad \text { weakly star } \quad L^{\infty}\left(0, T ; L^{2}(\Omega)\right) .
$$


Next we show the convergence of nonlinear term $\left|u_{k}\right|^{2} u_{k}$. By Sobolev inequality, we have

$$
\left.\left.\int_{\Omega}|| u_{k}\right|^{2} u_{k}\right|^{2} \mathrm{~d} x=\int_{\Omega}\left|u_{k}\right|^{6} \mathrm{~d} x \leq C\left\|u_{k}\right\|_{H_{0}^{1}(\Omega)}^{6} \leq C .
$$

Then there exists some function $\tilde{\chi} \in L^{\infty}\left(0, T ; L^{2}(\Omega)\right)$ such that

$$
\left|u_{k}\right|^{2} u_{k} \rightarrow \tilde{\chi} \quad \text { weakly star } L^{\infty}\left(0, T ; L^{2}(\Omega)\right) .
$$

Using (3.24) and Lemma 3.1.5 in [23], we get

$$
\tilde{\chi}=|u|^{2} u
$$

which concludes

$$
\left|u_{k}\right|^{2} u_{k} \rightarrow|u|^{2} u \quad \text { weakly star } \quad L^{\infty}\left(0, T ; L^{2}(\Omega)\right) .
$$

Now, using the convergence (3.18)-(3.20), (3.25) and (3.26) we can pass to the limit in (3.1) to obtain

$\left\langle u^{\prime \prime}, w_{j}\right\rangle+\left(\nabla u, \nabla w_{j}\right)+\left(u, w_{j}\right)+\left(u^{\prime}, w_{j}\right)-\left(u \log |u|^{2}, w_{j}\right)+\left(|u|^{2} u, w_{j}\right)=0, \forall j$.

Since the system $\left\{w_{j}\right\}_{i=1}^{\infty}$ is dense in $H_{0}^{1}(\Omega)$, we have

$$
\left\langle u^{\prime \prime}, v\right\rangle+(\nabla u, \nabla v)+(u, v)+\left(u^{\prime}, v\right)-\left(u \log |u|^{2}, v\right)+\left(|u|^{2} u, v\right)=0
$$

for any $v \in H_{0}^{1}(\Omega)$. That is to say $u$ satisfies the equation (1.1) in the weak sense.

In what follows, we check that $u$ satisfies the initial condition. From (3.18) and (3.19), we have

$$
u_{k}(0) \rightarrow u(0) \quad \text { weakly in } L^{2}(\Omega) .
$$

Since we have chosen $u_{k}(0)$ such that

$$
u_{k}(0) \rightarrow u^{0} \quad \text { strongly in } H_{0}^{1}(\Omega) .
$$

Therefore, we have

$$
u(0)=u^{0} .
$$

It can be inferred from (3.21) that

$$
\left\langle u_{k}^{\prime \prime}, w_{j}\right\rangle \rightarrow\left\langle u^{\prime \prime}, w_{j}\right\rangle \text { in } L^{\infty}(0, T),
$$

which implies that

$$
\left\langle u_{k}^{\prime}(0), w_{j}\right\rangle \rightarrow\left\langle u^{\prime}(0), w_{j}\right\rangle .
$$

Noting that

$$
u_{k}^{\prime}(0) \rightarrow u^{1} \quad \text { strongly in } L^{2}(\Omega),
$$

then we have

$$
u^{\prime}(0)=u^{1} .
$$

As a consequence of (3.27) and (3.28), the initial condition is satisfied. Therefore, the global existence of weak solutions to the problem (1.1) is established. Then the proof of Theorem 2.1 is complete. 


\section{References}

[1] J. D. Barrow and P. Parsons, Inflationary models with logarithmic potentials, Phys. Rev. D 52 (1995), 5576-5587.

[2] K. Bartkowski and P. Gorka, One-dimensional Klein-Gordon equation with logarithmic nonlinearities, J. Phys. A 41 (2008), no. 35, 355201, 11 pp.

[3] I. Bialynicki-Birula and J. Mycielski, Wave equations with logarithmic nonlinearities, Bull. Acad. Polon. Sci. Sér. Sci. Math. Astronom. Phys. 23 (1975), no. 4, 461-466.

[4] - Nonlinear wave mechanics, Ann. Physics 100 (1976), no. 1-2, 62-93.

[5] H. Buljan, A. Siber, M. Soljacic, T. Schwartz, M. Segev, and D. N. Christodoulides, Incoherent white light solitons in logarithmically saturable noninstantaneous nonlinear media, Phys. Rev. E (3) 68 (2003), no. 3, 036607, 6 pp.

[6] T. Cazenave, Stable solutions of the logarithmic Schrödinger equation, Nonlinear Anal. 7 (1983), 1127-1140.

[7] T. Cazenave and A. Haraux, Équations d'évolution avec non-linéarité logarithmique, Ann. Fac. Sci. Toulouse Math. (5) 2 (1980), no. 1, 21-51.

[8] — Équation de Schrödinger avec non-linéarité logarithmique, C. R. Acad. Sci. Paris Sér. A-B 288 (1979), no. 4, A253-A256.

[9] S. De Martino, M. Falanga, C. Godano, and G. Lauro, Logarithmic Schrödinger-like equation as a model for magma transport, Europhys. Lett. 63 (2003), no. 3, 472-475.

[10] K. Enqvist and J. McDonald, Q-balls and baryogenesis in the MSSM, Phys. Lett. B 425 (1998), 309-321.

[11] Y. Giga, S. Matsuiy, and O. Sawada, Global existence of two-dimensional Navier-Stokes flow with nondecaying initial velocity, J. Math. Fuid Mech. 3 (2001), no. 3, 302-315

[12] P. Gorka, Logarithmic Klein-Gordon equation, Acta Phys. Polon. B 40 (2009), no. 1, 59-66.

[13] _ Logarithmic quantum mechanics: existence of the ground state, Found. Phys. Lett. 19 (2006), no. 6, 591-601.

[14] _ Convergence of logarithmic quantum mechanics to the linear one, Lett. Math. Phys. 81 (2007), no. 3, 253-264.

[15] P. Gorka, H. Prado, and E. G. Reyes, Functional calculus via Laplace transform and equations with infinitely many derivatives, J. Math. Phys. 51 (2010), no. 10, 103512, 10 pp.

[16] - Nonlinear equations with infinitely many derivatives, Complex Anal. Oper. Theory 5 (2011), no. 1, 313-323.

[17] L. Gross, Logarithmic Sobolev inequalities, Amer. J. Math. 97 (1975), no. 4, 1061-1083.

[18] T. Hiramatsu, M. Kawasaki, and F. Takahashi, Numerical study of Q-ball formation in gravity mediation, Journal of Cosmology and Astroparticle Physics 2010 (2010), no. 6, 008 .

[19] W. Krolikowski, D. Edmundson, and O. Bang, Unified model for partially coherent solitons in logarithmically nonlinear media, Phys. Rev. E 61 (2000), 3122-3126.

[20] A. Linde, Strings, textures, inflation and spectrum bending, Phys. Lett. B 284 (1992), $215-222$.

[21] V. S. Vladimirov, The equation of the p-adic open string for the scalar tachyon field, Izv. Math. 69 (2005), no. 3, 487-512.

[22] V. S. Vladimirov and Ya. I. Volovich, Nonlinear dynamics equation in p-adic string theory, Teoret. Mat. Fiz. 138 (2004), 355-368.

[23] S. Zheng, Nonlinear Evolution Equations, Chapman \& Hall/CRC, Boca Raton, FL, 2004. 
Institute of Contemporary Mathematics

Henan University

Kaifeng 475004, P. R. China

AND

School of Mathematics and Information Science

HENAN UNIVERSITY

Kaifeng 475004, P. R. China

E-mail address: xiaosen_han@163.com 